\title{
SINGULAR PERTURBATION OF NONLINEAR TWO-POINT BOUNDARY VALUE PROBLEMS
}

\author{
H. S. NUR
}

Abstract. Under certain assumptions on $f\left(x, y^{\prime}, y, \boldsymbol{\epsilon}\right)$, this paper discusses the existence and asymptotic behavior of the solution of $\epsilon y^{\prime \prime}+f\left(x, y^{\prime}, y, \epsilon\right)=0$ with $y(0)=A$ and $y(1)$ $=B$.

Consider the equations

(1) $y^{\prime \prime}+f\left(x, y, y^{\prime}, \epsilon\right)=0$, with $y(0)=A$ and $y(1)=B$, where $x \in[0,1]$

(2) $f\left(x, y_{0}{ }^{\prime}, y_{0}\right)=0$, with $y_{0}(1)=B$.

Subtract (2) from (1) and obtain

(3) $\epsilon y^{\prime \prime}+f\left(x, y^{\prime}, y, \epsilon\right)-f\left(x, y_{0}{ }^{\prime}, y_{0}, 0\right)=0$.

Using the mean value theorem for several variables, we obtain

$$
\boldsymbol{\epsilon} y^{\prime \prime}+\boldsymbol{\alpha}(x, \boldsymbol{\epsilon})\left(y^{\prime}-y_{0}{ }^{\prime}\right)+\boldsymbol{\beta}(x, \boldsymbol{\epsilon})\left(y-y_{0}\right)=0,
$$

where $\alpha$ and $\beta$ are continuous functions in both $x$ and $\epsilon$, and $\alpha(x, \epsilon)$ is positive (or negative) on $[0,1]$.

Now we wish to impose certain conditions on $f\left(x, y^{\prime}, y, \epsilon\right)$ so that the above conditions on $\alpha$ and $\beta$ are satisfied. Such assumptions are: $f$ is $C^{\infty}$ in all variables, and $f_{y}$ and $f_{y^{\prime}}$ are positive.

Subtract $\left(\epsilon y_{0}{ }^{\prime \prime}\right)$ from both sides of (4), and let $y-y_{0}=u$; we obtain

$$
\epsilon u^{\prime \prime}+\alpha(x, \epsilon) u^{\prime}+\beta(x, \epsilon) u=-\epsilon y_{0}{ }^{\prime \prime},
$$

with the conditions $u(0)=A-y_{0}(0)$ and $u(1)=0$.

Since the function $y_{0}{ }^{\prime \prime}$ is independent of $\epsilon$, then obviously it is sufficient to consider the equation

$$
\begin{gathered}
\epsilon u^{\prime \prime}+\alpha(x, \epsilon) u^{\prime}+\beta(x, \epsilon) u=0, \\
\text { with } u(0)=A-y_{0}(0) \text { and } u(1)=0,
\end{gathered}
$$

i.e., if (6) is stable, so is (5).

If we let $u^{[k]}=\epsilon^{k}\left(d^{k} u / d x^{k}\right)$, then (6) becomes

$$
u^{[2]}+\alpha(x, \epsilon) u^{[1]}+\epsilon \beta(x, \epsilon) u=0 .
$$

Let $\omega_{1}(x) \quad(i=1,2)$ be the roots of $\omega^{2}+\alpha(x, 0) \omega=0$.

LemMa 1. For each $i(i=1,2)$ there exists an infinite number of functions $u_{i 0}, u_{i 1}, \cdots$ continuous and with continuous derivatives of all orders such that $u_{i 0}(x)$ does not vanish at any point of $[0,1]$, and 
if the functions $u_{i}(x, \epsilon)=\exp \left[(1 / \epsilon) \quad \int_{0}^{x} \omega_{i}(s) d s\right] \quad \sum_{j=0}^{m-1} u_{i j}(x) \epsilon^{j}$ are substituted in (7) for $u$, then the coefficients of $\exp [(1 / \epsilon)$ $\left.\int_{0}^{x} \omega_{i}(s) d s\right] \epsilon^{h}, i=1,2 ; h=0,1, \cdots, m$, vanish identically.

Lemma 2. The D.E. (7) has two linearly independent solutions:

$$
y_{i}(x, \epsilon)=u_{i}(x, \epsilon)+\epsilon^{m} E_{0}
$$

for any positive integer $m$ where $E_{0}$ is a bounded function.

The proofs of Lemmas 1 and 2 are in [1]. find

Now let $Y=c_{1} y_{1}+c_{2} y_{2}$ and apply the boundary conditions. We

$$
c_{1}=\frac{\left[A-y_{0}(0)\right] y_{2}(1)}{-\left[u_{20}(0) u_{10}(1)+O(\epsilon)\right]} \rightarrow 0, \quad \text { as } \epsilon \rightarrow 0,
$$

and

$$
c_{2}=\frac{y_{1}(1)\left(A-y_{0}(0)\right)}{u_{20}(0) u_{10}(1 ;)+O(\epsilon)}
$$

which is bounded as $\epsilon \rightarrow 0$.

Since $y_{2}(x, \epsilon) \rightarrow 0$ as $\epsilon \rightarrow 0$, we find that $Y(x, \epsilon) \rightarrow 0$ as $\epsilon \rightarrow 0$. Consequently the solution $u(x, \epsilon)$ of (5) goes to zero as $\epsilon \rightarrow 0$ which in turn shows that $y(x, \epsilon)$, the solution of $(1)$, goes to the solution $y_{0}(x)$ of $(2)$.

Remarks. Notice that the boundary condition $y_{\epsilon}(1)$ coincides with $y_{0}(1)$. However, if we assume $y_{\epsilon}(0)=y_{0}(0)$ instead we get $y(x, \epsilon) \rightarrow$ $y_{0}(x)+h(x)$. So it is necessary that the two solutions agree at 1 . Another point needs to be mentioned here. Since $y^{\prime}(x, \epsilon)$ may not be bounded in $\epsilon$ at $x=0$, this necessitates that we impose an extra condition on $f\left(x, y^{\prime}, y, \epsilon\right)$ or we would have some exceptions. But if it happens that $y(0, \epsilon)=y_{0}(0)$ and $y(1, \epsilon)=y_{0}(1)$, then $y^{\prime}(x, \epsilon)$ is bounded in $[0,1]$.

Now we shall apply this technique to the equation

$$
\begin{gathered}
\epsilon y^{\prime \prime}+y y^{\prime}-y=0, \text { with the conditions } \\
y(0)=A>0, \text { and } y(1)=B>A+1,
\end{gathered}
$$

whose unperturbed equation is

$$
y y^{\prime}-y=0,
$$

which has the solution $y_{0}(x)=x+B-1$.

So, subtracting (9) from (8), assuming $y_{\epsilon}-y_{0}=u$ and noticing that $y_{0}{ }^{\prime \prime}=0$, we obtain by using the mean-value theorem the following 
equation,

$$
\begin{aligned}
& \epsilon u^{\prime \prime}+y_{0} u^{\prime}+\left(y^{\prime}-1\right) u=0, \\
& \text { with } u(0)=A+1-B \text { and } u(1)=0 .
\end{aligned}
$$

According to Lemma 2, this equation has the two linearly independent solutions

$$
\begin{aligned}
& y_{1}=u_{1}(x, \epsilon)+\epsilon^{m} E_{0}, \\
& y_{2}=u_{2}(x, \epsilon)+\epsilon^{m} E_{0},
\end{aligned}
$$

where

$$
\begin{aligned}
& u_{1}=\sum_{0}^{m-1} u_{1 j}(x) \epsilon^{j}, \text { and } \\
& u_{2}=\left[\exp \left(-(1 / \epsilon) \int_{0}^{x} y_{0}(s) d s\right)\right] \quad\left[\sum_{0}^{m-1} u_{2 j}(x) \epsilon^{j}\right] .
\end{aligned}
$$

Thus, the general solution is, $Y=c_{1} y_{1}+c_{1} y_{2}$, and with the boundary conditions we obtain

$$
c_{1}=\frac{[A-B+1] y_{2}(1)}{\left[u_{20}(0) u_{10}(1)+O(\epsilon)\right]} \rightarrow 0 \quad \text { as } \quad \epsilon \rightarrow 0
$$

and

$$
c_{2}=\frac{y_{1}(1)(A-B+1)}{u_{20}(0) u_{10}(1)+O(\epsilon)} \text { which is bounded as } \epsilon \rightarrow 0 .
$$

Hence we find $Y(x, \epsilon) \rightarrow 0$ as $\epsilon \rightarrow 0$ which gives $y_{\epsilon}(x) \rightarrow y_{0}(x)$ as $\epsilon \rightarrow 0$.

\section{REFERENCE}

1. H. S. Nur, Singular Perturbation of Linear P.D.E., J.D.E. 6 (1969).

California State University, Fresno, California 93710 
